Cahiers de philosophie de l'université de

Levinas : au-delà du visible

\title{
La possession, la chose et le sujet : la théorie du besoin dans les Carnets de captivité
}

\section{Zinaida Sokuler}

\section{(2) OpenEdition}

1 Journals

Édition électronique

URL : https://journals.openedition.org/cpuc/862

DOI : $10.4000 /$ cpuc.862

ISSN : 2677-6529

Éditeur

Presses universitaires de Caen

\section{Édition imprimée}

Date de publication : 31 juillet 2012

Pagination : 197-214

ISBN : 978-2-84133-410-0

ISSN : 1282-6545

\section{Référence électronique}

Zinaida Sokuler, « La possession, la chose et le sujet : la théorie du besoin dans les Carnets de captivité ", Cahiers de philosophie de l'université de Caen [En ligne], 49 | 2012, mis en ligne le 07 juin 2018, consulté le 01 février 2023. URL : http://journals.openedition.org/cpuc/862 ; DOI : https:// doi.org/10.4000/cpuc. 862

\section{(c) (†) (8)}

Creative Commons - Attribution - Pas d'Utilisation Commerciale 4.0 International - CC BY-NC 4.0 https://creativecommons.org/licenses/by-nc/4.0/ 


\section{La possession, la chose et le sujet: la théorie du besoin dans les Carnets de captivité}

\section{La situation de la question du besoin}

Pour un chercheur qui étudie et interprète la pensée d'un grand philosophe ou d'un écrivain, une initiation à ses carnets privés et aux ébauches de ses conceptions a une signification toute particulière. Elle donne la sensation de l'intimité, comme si une certaine barrière était cassée et que l'on recevait des contacts plus proches et intimes avec le penseur, les idées et le monde intérieur que l'on essaie de comprendre. L'étude de carnets peut donner une expérience d'une grande force émotionnelle. Mais dans le cas des Carnets de captivité nous recevons aussi une expérience exceptionnelle, parce qu'il s'agit des notes prises dans des conditions où la proximité de la mort, l'humiliation et la souffrance, étaient devenues la constante de l'existence.

En général, pour comprendre un penseur il est important de savoir parmi ses idées lesquelles mûrissent plus tôt, lesquelles plus tard, et dans quelles conditions cela s'est passé. Nous voyons que Levinas a élaboré les idées du besoin et de la jouissance dans un camp de prisonniers. Un aperçu de la théorie du besoin dans le carnet 4 , quoique très laconique et sommaire, semble mûr déjà dans ses aspects principaux (par rapport à De l'existence à l'existant et à Totalité et Infini), tandis que le désir métaphysique n'y est présenté que sous la forme du désir érotique. La théorie lévinassienne de la jouissance prend donc sa forme dans les conditions de la captivité, c'est-à-dire dans des conditions de privation et de souffrance. Et c'est fort surprenant, bien sûr. Mais qu'est-ce que cela permet de mieux comprendre de Levinas et de ses idées? 
Rappelons d'abord que l'interrogation de la présence de Dieu ou de la trace de cette présence est toujours là dans les Carnets (il faudrait remarquer que cette question dans les conditions d'un camp de prisonniers était proche de celle d'une théodicée). On peut évoquer aussi tout le texte de L'expérience juive du prisonnier ${ }^{1}$. Il y a dans les Carnets des notes répétées comme: «L'essentiel: entendre l'appel de Dieu $[\ldots] »^{2}$, ou encore «[... L'essentiel n'est-il pas dans le fait que l'on souffre à la face de Dieu $[\ldots]{ }^{3}$. On trouve encore le thème du martyre, de la fidélité et de l'élection.

Martyre. Ce n'est pas seulement le sacrifice de sa vie. Ce qui importe, c'est la fidélité jusqu'à la mort. On n'ose pas dire que le malheur est à son comble, que l'abandon par Dieu est total et que l'heure de la malédiction est venue car on a encore le temps. Le martyre est au-delà de cette fidélité - est la fidélité dans la mort - là où il ne peut plus y avoir de miracle. Et en même temps le salut pour le présent et non pas une simple récompense dans l'avenir ${ }^{4}$.

Il s'agit dans cette dernière citation d'un problème qui semble crucial, car Levinas parle ici d'une situation dans laquelle il n'est plus possible d'attendre des signes de la présence de Dieu (en guise de sauvetage miraculeux). Cela explique que Levinas dans son œuvre cherche toujours quelque chose qui peut être l'indice de la dimension d'au-delà et qui est déjà là, avec nous. Plus tard il l'appellera «la trace $»^{5}$.

Dans L'expérience juive du prisonnier, nous lisons comment enfin

Les forces du mal reculaient partout. Le communiqué allemand n'enregistrait que des défaites. [...] Oui, Dieu a aimé Israël d'un amour éternel - oui, il nous a sauvés de la main de tous les tyrans $[\ldots]^{6}$.

Mais le problème consiste en ce que la main de Dieu n'est pas toujours visible si nettement. C'est pourquoi Levinas a écrit qu' «Être religieux - ce n'est pas croire à l'existence de Dieu - ce qui est une abstraction - mais avoir

1. E. Levinas, L'expérience juive du prisonnier, in Carnets de captivité, suivi de Écrits sur la captivité et Notes philosophiques diverses (= Euvres 1), R. Calin et C. Chalier (éd.), Paris, Grasset - IMEC, 2009, p. 209-215, surtout p. 213.

2. Carnets de captivité, p. 78.

3. Ibid., p. 68, voir aussi p. 180.

4. Ibid., p. 70. Voir aussi p. 82, 87 .

5. Ce thème de la trace est à peine ébauché dans les Carnets. On peut le présupposer seulement en voyant l'intérêt inattendu de Levinas pour Sherlock Holmes, quand il écrit «je fais un pas [...] je laisse une trace» (ibid., p. 188), ou « On agit toujours en laissant des traces. - Chose curieuse: pas de déduction: le déduit est immédiatement vu dans les indices» (p. 133). Cette note à propos de ce qui est immédiatement $v u$ dans la trace est bien significative.

6. L'expérience juive du prisonnier, p. 214. 
une question de Dieu [...]» ${ }^{7}$. Je peux comprendre cette question de Dieu seulement comme celle de la présence de Dieu, surtout dans la situation où

La force [est] dans son triomphe le plus brutal, dans ce triomphe qui fait douter de tout ce qu'on avait enseigné sur le Bien et le Mal, sur un monde gouverné par une Miséricorde. Quelqu'un l'a dit: c'est à croire que Dieu n'est pas bon ou qu'il n'est pas puissant ${ }^{8}$.

Le thème de «l'élection dans la souffrance» ne sera pas envisagé ici, car il s'agira de montrer par la suite que cette expérience n'épuise pas la question de la trace divine dans les Carnets. Maintenant il est nécessaire de souligner que le contexte théorique de la théorie du besoin est formé non seulement par le désir de quitter le climat de la philosophie heideggérienne, mais aussi par cette quête de la trace de Dieu.

\section{L'il y a comme plaie d'Égypte}

La théorie du besoin a été élaborée dans le carnet 4 et on voit plus tard dans De l'existence à l'existant et Totalité et Infini comment Levinas comprend les thèmes de la possession, de la chose, de la subjectivité et de l'il y $a-$ l'il y a étant une véritable entrée dans ce nœud de problèmes. Cette notion énigmatique doit donc maintenant être abordée.

Au lieu de la paire «l'être et le néant» Levinas propose une structure beaucoup plus complexe. Au sein de l'être, il distingue le «il y a» associé avec la nuit, d'une part, et l'être comme la lumière, d'autre part. L' ' il y $a=$ nuit de l'être ${ }^{9}$. Plus tard, dans la conférence "Les Nourritures" prononcée en 1950 au Collège philosophique, Levinas parlera des ténèbres («la plaie d’Égypte»), où se passe

[la] déshumanisation totale de la réalité [...] une présence anonyme, ni objective, ni subjective [...] où le moi perd sa fixité même. Il y $a$ <anonymat/ anonymement? $>$ de l'être ${ }^{10}$.

Dans De l'existence à l'existant, Levinas décrit l'horreur de l'il y a. L'horreur de l'il y a semble plus grande que celle du néant et de la mort. L'il y a impersonnel nous fait penser à l'origine mythique de l'être, mais

7. Carnets de captivité, p. 196.

8. L'expérience juive du prisonnier, p. 212.

9. Ibid., p. 103.

10. E. Levinas, "Les Nourritures", in Parole et Silence et autres conférences inédites au Collège philosophique (= Euvres 2), R. Calin et C. Chalier (éd.), Paris, Grasset - IMEC, 2011, p. 157. 
il vaut mieux penser à l'époque contemporaine, à l'époque des sociétés totalitaires, des exterminations massives satisfaisant des critères d'effectivité, des armements de destruction massive, impersonnels et indifférents comme l'il y a lui-même.

L'il y $a$, c'est une existence sans le sens, ou plutôt l'existence où l'absence du sens n'a aucune importance. C'est l'existence qui ne provoque aucune envie d'exister, ou plutôt qui est complètement indifférente à ce qui donne envie de continuer l'existence ou non. On comprend qu'il n'y a aucun espoir dans l'il y $a$. Non seulement il n'y a pas là d'espoir, mais il n'y a pas non plus de temporalité.

L'idée de l'être qui est égal à la nuit, de l'être si horrible, semble sans précédent. C'est l'être qui est la plaie d'Égypte même. On voit que cette idée est cristallisée dans un camp de prisonniers. À vrai dire, on l'avait toujours su, mais maintenant nous avons les témoignages entre les mains. La naissance d'une telle idée dans des conditions pareilles est fort compréhensible. Et en même temps ce n'est pas tout à fait compréhensible quand même. Étant monothéiste, Levinas adopte la doctrine de la création ex nihilo selon laquelle l'être est une créature de Dieu, y compris l'il y a. Pour la pensée européenne médiévale et pas seulement médiévale Ens et bonum convertuntur. L'être était considéré comme le don précieux, le don de Dieu (parfois comme le Dieu même, ou comme l'attribut de Dieu). Il n'en va plus ainsi pour Levinas. En effet, ce «don" peut être trop pesant, fatigant, horrible même.

La mention de la «plaie d'Égypte» peut nous faire penser qu'il s'agit ici du châtiment de Dieu, mais ce n'est pas vrai. L'il y a n'est pas un sort pour les pécheurs. Il y a l'il $y$ a tout simplement. Levinas ne dirait jamais que tous ceux pour qui l'être devient plus terrible ou plus pénible que la mort sont des pécheurs. On voit que selon Levinas Dieu a créé l'être qui n'a pas de sens. C'est une dimension fondamentale de l'ontologie lévinassienne.

Dans la nuit de l'il y a aucune dimension axiologique n'est à considérer. La théorie du besoin nous montre justement comment surgit cette dimension. Cela se passe grâce à la jouissance, le besoin étant une condition de satisfaction et de jouissance. Donc, le sens surgit dans l'être avec la jouissance humaine, ou, pour le dire autrement, avec l'être humain qui se pose et tend à la jouissance. La jouissance devient donc un trait ontologique du sujet humain, opposée à la nuit de l'il y a dénuée de sens. Cela a beaucoup de conséquences pour la compréhension du sujet humain et on voit que la théorie du besoin dans le carnet 4 est en même temps une ébauche d'une constitution du sujet. 


\section{L'apparition du sujet}

Il est manifeste que la compréhension du sujet est une préoccupation importante de Levinas dans les Carnets. En opposition à la philosophie classique, il veut mettre en lumière un sujet qui porte en lui-même la différenciation, le dynamisme et la dialectique. Levinas écrit cela très nettement dans les Carnets. Il reste à voir comment ce changement de l'idée du sujet est lié avec la recherche des traces de la présence de Dieu. Tout d'abord dans les Carnets, Levinas cherche la constitution d'un sujet à partir de l'il y $a$. Le sujet apparait, se pose contre l'il y $a$. Il s'agit de son mode d'individuation qui rompt avec l'anonymat de l'il y a.

Il serait peut-être intéressant de noter qu'Hermann Cohen, dans sa Religion de la raison tirée des sources du judaïsme, se heurte à un problème analogue: l'individuation et la constitution du sujet autonome. Cohen réfléchit comment un individu, un «Moi» apparaît à partir du sein mythique et anonyme du genre ou de la race. Il conclut que le moyen méthodique pour la découverte de l'individualité est un péché, l'individu étant défini comme une source indépendante de ses péchés, c'est-à-dire comme responsable et coupable.

On trouve plus tard une idée analogue chez Levinas (que l'identité du Moi est constituée par la responsabilité et la culpabilité), mais pas ici. Hermann Cohen est mentionné juste pour souligner qu'avec la théorie lévinassienne du besoin nous sommes à un niveau tout à fait différent. Chez Cohen, il s'agit du monde social avec l'impératif biblique «Tu aimeras ton prochain comme toi-même». Mais pour Levinas, c'est justement à partir des ténèbres impersonnelles de l'il y a que le sujet fait son apparition. Levinas insiste sur cette idée très nettement dans les Carnets et dans De l'existence à l'existant. Cette doctrine de l'apparition du sujet du sein de l'il y a ne se trouve plus dans les œuvres ultérieures. Dans Totalité et Infini, elle est remplacée par l'idée du psychisme et de la séparation, bien qu'il parle dans Autrement qu'être ou au-delà de l'essence

[du] Bourdonnement de l'il y $a-[\ldots]$ intolérable à un sujet qui se libère comme sujet [...] Ambiguité du sens et du non-sens dans l'être, le sens tournant en non-sens ${ }^{11}$.

N'y a-t-il rien de plus tragique que cette doctrine issue des années de captivité, où le sujet se pose contre la nuit de l'il y $a$ ? On n'a pas encore de morale, ni de péché, ni de responsabilité, on n'a pas de sens. Cela veut dire que les valeurs et les devoirs de la morale ne sont pas encore construits

11. Autrement qu'être ou au-delà de l'essence, La Haye, Martinus Nijhoff, 1974, p. 254. 
théoriquement. Il s'agit jusqu'ici du sujet jouissant de la vie dans tout son égoïsme innocent. Les devoirs et la transcendance restent encore à constituer. Mais la construction commence par l'intermédiaire du besoin, à partir du sujet jouissant. À ce niveau ontologique, le sujet ou le Moi apparaît en obtenant la maîtrise sur l'être par ses besoins et ses appétitions:

L'appétition est une autre manière de maîtrise sur l'être - voila mon idée centrale. Donc l'appétition doit être envisagée dans la perspective même de la subjectivité. C'est le même problème : comment - dans quel sens - l'anonymat de l'il y a [...] a-t-il une structure subjective - dans quel sens il peut y avoir commencement et sujet ${ }^{12}$ ?

\section{Le Moi de la jouissance}

La catégorie du besoin nous est apparue dans le carnet 4 comme étant étroitement liée à la notion de la chose : "La relation avec les choses - se réfère au besoin ${ }^{13}$. Levinas ajoute: «[...] besoin catégorie spéciale de la relation avec des choses ${ }^{14}$. En effet, les choses, souligne Levinas,

ce n'est pas le maniable, mais l'utilisable. L'utilisable - n'est pas ce qui est nécessaire à l'action [...] Les choses sont nécessaires pour être ${ }^{15}$.

Les choses, ou plus exactement les relations avec des choses, deviennent chez Levinas un certain existential (au sens heideggérien). La compréhension de la chose comme un moment très important de l'expérience existentielle est une thèse qui apparait très tôt dans la pensée de Levinas. Voici une note datée de septembre 1937:

Argent - abstraction. [...] Les choses ont une valeur de choses et non pas d'argent. User jusqu'à la corde. Pas avarice ${ }^{16}$.

On voit ici une expression du lien intime entre un être humain et une chose, comme si cette chose devenait l'une de ses parties. Ou, plutôt, comme si la chose avait son sens à elle. Il faut rappeler que la philosophie n'a presque jamais approuvé une telle attitude par rapport aux choses. Elle a tâché de libérer l'être humain de l'attachement aux choses, de l'attachement à l'extériorité en général, et c'est pourquoi si souvent la chose a été envisagée comme une illusion cachant l'être vrai ou cachant le sujet de lui-même.

12. Carnets de captivité, p. 121.

13. Ibid., p. 117.

14. Ibid., p. 118.

15. Ibid., p. 117-118.

16. Ibid., p. 51 . 
Il faut dire d'abord que Levinas n'est pas tout à fait étranger à cette tradition de pensée. On le voit surtout dans ses ébauches de romans qui nous fournissent un supplément intéressant à la théorie du besoin. Que de descriptions impressionnantes il nous offre! Par exemple:

Les réfugiés qui partent avec des charrettes les plus absurdes - incapable de partir seul, de rester seul. Comme la limace qui porte sa coquille ${ }^{17}$.

Ou encore: «Le pillage des vitrines - les gens qui emportent ce qui n'a aucun sens $[\ldots]{ }^{18}$. Les personnes se comportent comme si les choses pouvaient garder des résidus du sens collés à elles quand le monde est cassé et perd son sens.

En parlant de la draperie sociale et officielle qui tombe avec la défaite, Levinas montre que cela était accompagné par ce qu’il nomme «la dégradation des choses $»^{19}$.

Les draperies qui tombent dans ma scène d'Alençon concernent aussi les choses. Les choses se décomposent, perdent leur sens: les forêts deviennent arbres [...] je ne veux pas simplement parler de la fin des illusions; mais plutôt de la fin du sens. [...] [Et Levinas note pour lui-même] Creuser cette idée de la "perte de sens» par les choses ${ }^{20}$.

Cette perte du sens, elle semble bien compréhensible si les choses ne sont que des signes sociaux. Une fois les structures sociales cassées, les choses perdent leurs sens. Donc, les choses n'ont-elles pas de sens à elles? Dans l'essai Captivité, Levinas écrit que, si paradoxal que cela puisse paraître, la situation du prisonnier est aussi celle d'une libération. Les prisonniers «ont connu [...] une liberté insoupçonnée» ${ }^{21}$. En expliquant ce que cela signifie, Levinas mentionne

un dépouillement qui rendit le sens de l'essentiel. [...] plus rien de strictement privé. $[\ldots]$ Restait le lit: trois mètres cubes $[\ldots]^{22}$.

En cette situation pénible, Levinas souligne encore:

On possédait. Mais la propriété n'était pas votre maître [...] nous avons découvert qu'on n'en mourait pas. Nous avons appris la différence entre avoir et être. Nous avons appris le peu d'espace et le peu de choses qu'il faut pour vivre ${ }^{23}$.

17. Ibid., p. 104.

18. Ibid., p. 135 .

19. Ibid., p. 136.

20. Ibid., p. 132.

21. Captivité, in Euvres 1, p. 201-202.

22. Ibid., p. 202.

23. Ibid., p. 202-203, voir aussi p. 70. 
Cela ressemble au raisonnement philosophique tenu depuis l'Antiquité. Mais au lieu de moraliser à propos des fausses valeurs matérielles, Levinas élabore sa conception du besoin et de la jouissance issue du besoin. On ne peut pas le dire plus exactement et plus expressivement que Levinas le fait lui-même: «Flâner, aller, revenir sur ses pas. Le bonheur de vivre n'est pas le bonheur d'être. L'être est cadavre $»^{24}$.

Il s'agit du surgissement du Moi dans la jouissance liée aux choses: «la fin du chaos = séparation de la lumière et de l'obscurité = surgissement du Moi. La jouissance $=$ vie d'un Moi ${ }^{25}$. Ou, comme Levinas le formule dans la conférence «Les Nourritures» déjà mentionnée ci-dessus : «La jouissance est exclusivement mienne, elle $\mathrm{m}^{\prime}$ isole ${ }^{26}{ }^{2}$. La jouissance est un noyau d'individuation. Cette idée est maintenue dans les travaux ultérieurs. Notons: la jouissance et pas la mort! La mort, n'isole-t-elle pas selon Levinas? Il se peut que pour Levinas la mort lie surtout un individu au sort de son peuple ou de l'espèce humaine en général.

Donc les choses gardent une importance ontologique chez Levinas, la jouissance étant liée avec les choses. Dans les travaux ultérieurs, les choses gagnent encore en importance parce qu'elles peuvent être présentées à l'Autrui pour satisfaire ses besoins.

Les choses gardent toute leur signification parce que, premièrement, Levinas ne veut pas reconnaitre une représentation (ou la connaissance en général) comme une forme primordiale du rapport envers le monde extérieur. Il veut mettre en lumière un rapport plus pesant, plus charnel dans lequel le sujet ne peut pas s'écarter du monde extérieur. Il dévoile un sujet engagé dans l'extériorité. Deuxièmement, Levinas ne veut pas d'un sujet immuable, autonome et clos, tout au contraire. Donc, il doit mettre en évidence que les choses sont nécessaires pour le sujet.

Les dangers liés à cette attitude envers les choses, Levinas les dit en termes de possession en régime capitaliste et en régime socialiste. Il note:

Posséder dans le monde qui n'est pas socialiste - c'est être asservi par ce qu'on possède. [...] Dans le monde socialiste posséder par le travail - sans lutte d'où possession sans être possédé - affranchissement à l'égard des choses. Les choses deviennent comme l'air - présentes - l'être devient un présent ${ }^{27}$.

Sauf erreur, Levinas n'a pas répété plus tard de pareils propos à propos du socialisme. A-t-il idéalisé le monde socialiste selon l'esprit du temps? Peut-être, mais cela n'a pas grande importance en ce qui concerne sa théorie

24. Carnets de captivité, p. 69.

25. Notes philosophiques diverses, in Euvres 1, p. 243.

26. "Les Nourritures», p. 162.

27. Carnets de captivité, p. 115. 
du besoin, parce que les analyses qui viennent ensuite nous rappellent que ce monde socialiste est un monde du présent et non pas celui de l'avenir: «Le monde messianique est encore au-delà du monde socialiste ${ }^{28}$.

Cela signifie que pour Levinas des problèmes tels que l'aliénation, l'assujettissement ne peuvent pas être résolus au seul niveau social et par des moyens purement sociaux, sans la transcendance, sans la dimension d'au-delà. Le trait particulier de la pensée lévinassienne, le trait paradoxal, est que c'est la théorie du besoin comme construction ontologique qui doit mener au seuil de cet «au-delà » ${ }^{29}$.

Il faut souligner les moments principaux de cette construction que Levinas a repris pendant toute sa vie. Premièrement, cette construction part du Moi se posant, jouissant, quasi autonome. Levinas part de l'ego avec son égoïsme, c'est-à-dire de sa façon de maîtriser l'être en se satisfaisant. (Par ce trait la philosophie lévinassienne est toute proche de la construction conceptuelle de Rosenzweig qui part du Soi obstiné, solitaire, se posant contre tout.) Mais, deuxièmement, Levinas tente de montrer en même temps le défaut de la façon dont l'ego se pose afin de souligner ainsi la nécessité de cette dimension d'au-delà.

Cela est attesté dans le carnet 4 avec l'élaboration plus détaillée du besoin et de son accomplissement.

\section{La lumière comme mode d'être}

Il a déjà été montré que le besoin est une relation avec l'extérieur. Levinas l'explique en termes d'espace, d'intervalle, de vide et de lumière. L'intervalle, la distance séparent le besoin de son accomplissement, et c'est pourquoi la distance est la condition même de la jouissance. Par exemple, on respire l'air à chaque instant et dès qu'on a de l'air à respirer (il n'y a pas d'intervalle) on n'en jouit pas, on respire et c'est tout.

«L'intervalle» (ce mot que Levinas utilise souvent) a beaucoup de connotations différentes. C'est d'abord un vide. Un vide qui n'est pas le néant. Comme Levinas l'explique dans la conférence «Les Nourritures», le vide résulte de la négation inaccessible et impossible de l'il y $a$ :

Il y a qu'il est impossible de nier qui réapparaît derrière chacune des négations que l'on en tente. [...] La lumière est, précisément, par rapport à l'il y a une négation de ce qui ne peut être nié. Elle vide ${ }^{30}$.

28. Ibid.

29. Levinas n'aimait pas le mot ontologie. Mais il est possible de l'utiliser ici, parce qu'avec la théorie du besoin nous ne sommes pas au-delà, mais justement là, dans l'être.

30. «Les Nourritures», p. 157-158. 
C'est-à-dire que le vide surgit comme une étape sur la voie menant de l'il y $a$ à l'être rempli de sens. Le vide, à la différence de l'il y $a$, n'est pas horrible. Vidé de ténèbres grâce à la lumière, il est simplement vide. Le vide émerge comme une place à remplir, et c'est pourquoi le vide et l'intervalle ont aussi la signification d'une source (ou de la condition) de la liberté, de l'inattendu et de l'imprévisible.

L'intervalle signifie de même une certaine rupture, grâce à laquelle la chose désirée n'est pas le sujet, mais est extérieure au sujet. Cela veut dire que l'intervalle indique la différence entre l'accomplissement du besoin et l'idée dans la conscience du sujet. L'intervalle montre qu'il y a une frontière entre l'intériorité et l'extériorité, que le monde est hors du sujet. Et le sujet fait des efforts constants pour le maitriser, pour s'y poser.

Dans les remarques du carnet 4, Levinas parle souvent de l'espace. Les relations avec des choses, le besoin et son accomplissement, tout cela se passe dans l'espace et dans le présent. Levinas lie l'espace avec le présent et oppose ainsi l'espace et le temps, le temps (ou l'avenir) étant réservé pour une dimension d'au-delà ${ }^{31}$.

Bien que la distance s'associe facilement avec l'espace, il semble que dans ces remarques l'espace joue plutôt un rôle de métaphore. Plus tard Levinas lui-même n'utilisera plus cette opposition «l'espace et le temps" et la remplacera par l'opposition «synchronie et diachronie». Il faut noter que «diachronie» n'est pas le synonyme de l'avenir. Il y a là une différence importante, car la diachronie sera liée avec l'au-delà, alors que l'avenir ne sera plus lié avec l'au-delà. L'avenir peut être lié même avec l'il $y a$, car on ne peut jamais dire que «le malheur est à son comble», l'avenir pouvant être «la guerre de Gog et Magog ${ }^{32}$.

Remarquons aussi que des notions telle que le "chez soi», «le lieu», "l'habitation" (élaborées en détail plus tard), bien qu'ayant des connotations spatiales, sont en fait des notions ontologiques et non pas spatiales (j'utilise le mot «notion», mais il vaudrait peut-être mieux parler ici d'existentiaux au sens heideggérien).

Levinas explique que dans sa théorie du besoin, à l'opposé de celle de Platon, le besoin n'est pas envisagé comme un signe d'un manque, comme quelque chose de négatif seulement, mais comme un gage de la jouissance et

31. "Tout l'espace est donné dans la position. Le temps est toujours à venir" (Carnets de captivité, p. 120).

32. Voir la lecture talmudique «Au-delà du souvenir» dans E. Levinas, À l'heure des nations, Paris, Minuit, 1988, particulièrement p. 96-98. Levinas mentionne ici la possibilité des «[...] nouvelles épreuves d'Israël, dont la fin est peut-être toujours trop tôt proclamée [...]. Israël - ou l'humanité de l'Humain - n'a pas encore fini de constituer ses souvenirs. La coupe des souffrances d'Israël n'est pas encore vidée» (p. 97). 
du bonheur. La jouissance étant un lieu d'individuation, le souci de Levinas est de montrer des limitations internes du sujet qui se trouve posé de cette façon.

Cette limitation, d'où résulte-t-elle? Résulte-t-elle de l'encombrement du sujet, qui est déterminé par son propre passé qu'il traîne comme des bagages $^{33}$ ? Là nous revenons au problème de la dépendance humaine par rapport aux choses. En effet, tant de choses sont superflues! C'est le thème constant de la philosophie morale. Mais ce n'est pas cela qui est essentiel pour Levinas. À propos d'un sujet encombré, il écrit seulement que «Tout ce qui est - est là - et cependant il est encore libre à l'égard de toutes choses ${ }^{34}$. C'est-à-dire que pour Levinas il existe toujours un intervalle ou la possibilité de rompre les chaînes et de se donner la liberté.

Le sujet du besoin est limité par sa propre façon de maîtriser l'être, et c'est justement cela que Levinas tente de mettre en évidence. Cette façon est celle de l'acte de manger. Les métaphores telles que «manger» et «mordre» ont fait leur apparition dans les Carnets. Elles sont assez inattendues dans un texte philosophique, mais fort compréhensibles chez un prisonnier vivant dans des conditions de privation. L'idée est fort nouvelle et même révolutionnaire. Il s'agit de mettre en lumière que l'attitude principale du sujet envers le monde est de "manger ${ }^{35}$. Flâner, respirer, se promener, écouter du jazz, lire un livre de philosophie - c'est manger quelque chose d'appétissant (ou, peut-être, bon pour la santé?) qui m’est donné dans le monde et par qui le monde m'est donné:

[...] le sujet a aussi la maîtrise de l'être en le mangeant. Et c'est en tant qu'aliment que l'être est à intervalle et est monde - d'où possibilité de propriété - de possession ${ }^{36}$.

Ainsi le monde compris comme une nourriture est le monde extérieur, indépendant de ma conscience. Sinon il ne pourrait pas être appétissant (ou bon pour la santé). Le sujet l'utilise en le «mordant»:

[...] l'idée de mordre sur et de se revigorer à la morsure. Ce n'est pas l'idée chimique de l'assimilation - mais l'idée de maîtrise sur l'être - de subjectivité37.

En utilisant une chose extérieure et le monde en général, le sujet abolit l'intervalle entre lui-même et la chose (le monde). Cependant, "voir » c'est aussi «manger»:

33. Carnets de captivité, p. 120.

34. Ibid., p. 121.

35. On peut comprendre ici le sujet comme un homme en général ou comme le sujet de la philosophie classique - ce serait vrai dans les deux cas.

36. Carnets de captivité, p. 121.

37. Ibid. 
La vision et la connaissance elles-mêmes sont conçues chez moi comme couchées dans le lit du besoin qui est la catégorie première de l'extérieur ${ }^{38}$.

Le voir aussi, comme la connaissance, peuvent être conçus sur le modèle du "manger», parce qu'ils présupposent une distance entre le sujet et l'objet et l'éliminent en réduisant l'objet indépendant de moi à ma représentation. Cette attitude de «manger», Levinas ne la désapprouve pas. Il la décrit comme un fait de l'être humain. On peut dire que c'est un fait ontologique et on peut rappeler aussi des versets bibliques (Genèse 1. 28-29) ${ }^{39}$ où l'on peut voir l'origine de cette attitude.

La vision, tout aussi comme l'intervalle et l'accomplissement, présupposent la lumière. Dans les Carnets, il y a une grande quantité de notes consacrées spécialement à la lumière. Dans De l'existence à l'existant, il y a un paragraphe nommé «La lumière». Dans la conférence «Les Nourritures », Levinas parle aussi en détail de la lumière. Or on sait que la notion, ou plutôt l'image de la lumière, a toujours joué un rôle remarquable dans la philosophie. Dès la République de Platon, la lumière s'associe avec la vérité (et par là - avec la connaissance) et avec la présence de Dieu. Par un geste vraiment révolutionnaire, Levinas change ces associations. Les remarques concernant la lumière sont courtes, peu développées dans les Carnets, voire énigmatiques. On peut les comprendre, ou les déchiffrer, grâce à l'exposé développé dans la conférence «Les Nourritures ». Ce thème est donc important pour Levinas dès le commencement.

Pour Levinas, la lumière est liée avec l'espace. La lumière n'est pas l'espace bien sûr, mais peut-être est-elle un milieu où s'étend l'espace, où des besoins atteignent leur accomplissement et où la connaissance s'empare de son objet. Tout cela se passe dans la lumière:

Toute jouissance suppose lumière. C'est par la lumière que les appétitions sont autre chose que le besoin. Intervalle de la lumière ${ }^{40}$.

\section{Comme Levinas l'expliquera dans la conférence «Les Nourritures»:}

La lumière est un mode d'être et non pas un quelque chose: ni objet, ni sujet. [...] [Et un peu plus haut] Nous ne voyons pas la lumière, mais dans la lumière ${ }^{41}$.

38. Carnets de captivité, p. 118.

39. Où, s'adressant à l'homme et à la femme qu'il vient de créer « Dieu leur dit [...] remplissez la terre et l'assujettissez et dominer sur $[\ldots]$ ».

40. Carnets de captivité, p. 126.

41. «Les Nourritures», p. 158. 
Donc la lumière est dans un sens le principe même de l'extériorité: «Dans l'éclair de la lumière - origine de l'extériorité» ${ }^{42}$. Mais, d'un autre côté, étant si étroitement liée avec la connaissance et la jouissance qui «mangent» leur objet, la lumière devient chez Levinas l'intériorité: «L'idée de lumière $=$ idée d'immanence ${ }^{43}$. Il n'y a pas ici de contradiction. La lumière devient le principe même de cette transformation de l'extériorité en intériorité que le sujet et la connaissance accomplissent toujours. En outre, l'il y a étant lié avec l'absence du sens, la lumière est liée avec le sens : "Tout sens est question de lumière ${ }^{44}$. Cela semble assez évident dans la tradition philosophique qui associe toujours la compréhension et le sens avec la lumière. Et enfin, quand on voit le sens on parle toujours de la clarté.

On constate en somme que la lumière devient chez Levinas un terme entrant dans différentes oppositions en recevant une valeur fort ambivalente. Dans l'opposition «la nuit (il $y a$ ) / la lumière», la lumière possède un pôle positif. Elle est la condition de tout ce qui transforme la nuit de l'il y a en notre monde. Mais c'est exactement ce qui nous montre l'infériorité de la lumière. Dans l'opposition «là / au-delà», la lumière se situant du côté du «là» reçoit une valeur inférieure. La lumière s'associe avec l'immanence, la nourriture et - plus tard - avec le pouvoir. Cela veut dire que la lumière ne peut plus être associée avec le divin, le parfait, l'au-delà. Nous voyons ici la dégradation profonde de l'idée de la lumière. Il me semble que pour Levinas dans l'idée de la lumière sont réunis tous les traits négatifs que porte selon lui la tradition philosophique européenne. Mais Levinas n'écrit pas sur l'idée de la lumière, il écrit surtout sur la lumière même comme un fait ontologique. On peut donc dire qu'outre la nuit de l'il y a qui a une valeur négative, l'être inclut la lumière qui n'a qu'une valeur ambivalente. Et nous voyons ainsi que pour Levinas, en dépit de sa théorie de la jouissance, sa vision de l'être est fort pessimiste.

La lumière donc n'est plus un indice de la présence de Dieu comme elle a été dans la tradition philosophique. Et Levinas cherche toujours des indices nouveaux. Ici on peut m'objecter que pendant les années de captivité Levinas a trouvé cette confirmation dans la douleur et la souffrance même, car dans «la douleur [...] se fait ma passivité au sein de Dieu et mon élection ${ }^{45}$. Il semble quand même que cette réponse n'est pas complète et que Levinas, dans les Carnets, a cherché aussi une autre réponse. Il s'agit de la voie vers Autrui, bien sûr.

42. Carnets de captivité, p. 127.

43. Ibid., p. 160.

44. Ibid., p. 122.

45. Ibid., p. 154. 


\section{Le besoin et l'eros}

Levinas écrit souvent dans les Carnets que la destinée du sujet est la solitude. Cela est bien compréhensible, car le sujet ne sait que «manger» tout ce qu'il rencontre dans le monde en transformant l'extériorité en intériorité. Et pour la solitude, il se prive ainsi de la possibilité de s'en débarrasser.

Puis, le Moi du besoin qui découvre son soi dans la jouissance ne peut être à soi, car il dépend de ses besoins. En possédant, il est possédé. Il se perd ainsi toujours. Levinas écrit que «le moi maître du soi - ne s'absorbe pas dans le soi. Le moi est le maître d'un soi qui tout en étant soi n'est pas soi» ${ }^{46}$. Le moi ne coïncide pas avec le soi, il n'a pas d'identité stable.

Le retour du moi sur soi dans la jouissance [est] une réconciliation du moi avec le soi - découverte du soi par le moi - le moi se suffisant. Dans la satisfaction du besoin, il y a donc une plénitude de l'être ${ }^{47}$.

Levinas avoue cela, mais en même temps dit que «le moi dans la lumière n'a pas la répétition de l'identité. Il est dehors c'est-à-dire il n'est pas moi. Il existe sans être par lui-même ${ }^{48}$. Ainsi donc le sujet du besoin ne possède pas bien des traits du sujet classique, et ce n'est pas l'échec de la conception lévinassienne, c'est au contraire sa réussite, car les défauts du sujet du besoin mettent en relief la condition de l'existence humaine. Tout à la fois, ces manques servent à préparer le terrain pour le pas suivant dans la construction lévinassienne.

Outre la théorie du besoin, Levinas développe en effet dans les Carnets sa théorie de l'amour, qui est aussi opposée à la théorie platonicienne que celle du besoin.

La possession - c'est la main qui prend, mais c'est aussi le bras qui étreint. [...] La transformation de l'idée de possession. La possession - de la main qui prend. Celle de l'étreinte est de l'Eros ${ }^{49}$.

Pour sa théorie de la sexualité, où la dualité est essentielle, Levinas explique:

La sexualité. Conception qui permet de dépasser le problème «égoïsme altruisme». Puisque l'ego ne se définit pas en dehors de l'amour chez moi. Sexualité constitutive de l'égoïté. Rupture avec la conception antique de l'amour ${ }^{50}$.

46. Carnets de captivité, p. 123.

47. Ibid., p. 121.

48. Ibid., p. 127.

49. Ibid., p. 125 .

50. Ibid., p. 114 . 
C'est en exposant la théorie du besoin que Levinas explique la particularité du désir érotique:

Ce qui distingue le besoin de l'eros - c'est que le besoin est un intervalle franchi où la dualité disparaitt. [...] Dans l'eros c'est la dualité qui est la jouissance même. L'intervalle n'est pas seulement franchi - il est toujours à franchir ${ }^{51}$.

C'est là, dans sa théorie de l'amour, que Levinas parle du plaisir de l'Autre et de la douleur de la douleur de l'Autre:

Pour l'analyse de l'eros. Les sentiments indirects: souffrir de la souffrance d'autrui, se réjouir de ses joies. Réflexion sui generis. Et caractère infini de cette réflexion ${ }^{52}$.

Il me semble qu'on peut discerner dans ces analyses une genèse de l'idée de la substitution. Quoi qu'il en soit, en parlant de ce par quoi sa philosophie "diffère de la philosophie de Heidegger», Levinas souligne que "c'est l'importance de l'Autre. Eros comme moment central " ${ }^{53}$. L'Autre et l'eros vont donc ensemble au commencement de l'œuvre lévinassienne. En conséquence, on peut dire que, dans une première ébauche de la philosophie lévinassienne, Autrui est une bien-aimée. La théorie du besoin sert à donner la description ontologique de ce Moi qui a le désir érotique. Cette description montre que son identité n'est que le manque d'identité et que son indépendance n'est en réalité que dépendance. Ce moi est solitaire. Mais l'être est structuré par la dualité des sexes, et cela donne une possibilité de relation qui n'est pas celle du «manger», et qui sauve le sujet.

Un trait non moins remarquable de la conception développée dans les Carnets est que Levinas envisage l'eros comme l'origine du social. Par exemple: "Chez moi la collectivité a une dialectique sexuelle à sa base" 54 . «Amour-lutte. Origine du social» ${ }^{55}$.

Eros à la base du social - ce qui ne veut pas dire que le social s'explique par l'inférieur et qu'il n'y a rien de nouveau dans la société - Mais le social est déjà dans l'être ${ }^{56}$.

Tout cela nous fait penser qu'il y a dans les Carnets une dépendance vis-à-vis de la théorie de l'amour de Franz Rosenzweig. Selon Rosenzweig,

51. Ibid., p. 120.

52. Ibid., p. 70. Voir aussi p. 122.

53. Ibid., p. 134.

54. Ibid., p. 142

55. Ibid., p. 171. Voir aussi p. 172.

56. Ibid., p. 195. 
l'amour charnel, qui est, comme on sait, plus fort que la mort, devient une relation unique découvrant l'Autre comme Autre. L'amour est premièrement un signe de l'amour de Dieu. Rosenzweig souligne que le Cantique des Cantiques ne trouve pas sa place dans l'Écriture par hasard. L'amour charnel selon Rosenzweig est vraiment l'expérience de la présence divine. Il écrit:

Comme parabole, la parabole de l'amour traverse toute la Révélation. C'est la comparaison qui revient sans cesse chez les Prophètes. Mais il faut que ce soit plus qu'une parabole. Et elle l'est quand elle apparaît sans un "cela signifie", sans renvoi par conséquent à ce dont elle doit être la parabole. Il ne suffit donc pas que le rapport de Dieu à l'homme soit présenté à travers la parabole de l'amant et de l'aimée; la parabole de Dieu doit contenir immédiatement le rapport de l'amant à l'aimée, il faut qu'il y ait le signifiant sans la moindre allusion au signifié. Et c'est ainsi que nous le découvrons dans le Cantique des cantiques. Il me semble que le lecteur soit placé là devant le choix ou d'admettre le sens "purement humain", purement charnel et se demander alors par quelle erreur étonnante ces pages sont entrées dans la parole de Dieu, ou de reconnaître que là, précisément dans le sens purement charnel, est enfouie immédiatement, et pas "seulement" sous forme de parabole, la signification plus profonde ${ }^{57}$.

Ce raisonnement signifie que l'amour charnel n'est pas pour Rosenzweig (seulement) la parabole du rapport de Dieu à l'être humain, mais que l'amour charnel lui-même, en toute sa signification littérale, est la manifestation principale du fait que Dieu aime ses créatures. En recevant ce don miraculeux de l'amour sexuel, l'être humain comprend qu'il est aimé et élu par Dieu. Il commence d'aimer Dieu en réponse à son amour. Et c'est de la plénitude de son bonheur que l'homme commence à tenir le commandement de Dieu et à aimer son prochain. La vie d'une famille, de la nation, de la société paraît chez Rosenzweig comme un résultat de l'existence de l'amour. C'est dire que l'amour sexuel est pour Rosenzweig une base du social. Levinas est ainsi assez proche de Rosenzweig dans sa théorie de l'eros. Pour lui aussi l'eros donne le principe menant «au-delà». Cela signifie que l'eros, dans la genèse de l'œuvre d'Emmanuel Levinas, est une manifestation de la présence de Dieu.

Un témoignage indirect en faveur de cette supposition peut être trouvé dans l'attention que Levinas prête aux lettres de Léon Bloy. Ce que Levinas met en évidence dans ces lettres est intéressant, par exemple: «Pour Léon Bloy l'existence sensible dans son ensemble est un symbole transcendant. [...] Quelques exemples: le sexe de la femme - tabernacle du Christ $[\ldots] »^{58}$.

57. La traduction française citée est celle de S. Habib, Levinas et Rosenzweig: Philosophies de la Révélation, Paris, PUF, 2005, p. 220-221.

58. Carnets de captivité, p. 153. 
Cependant on trouve dans le carnet 7 une note qui semble contredire cette supposition:

La transcendance que je pose à la base de ma $\varphi$ - c'est $<$ sic $>$ ni la transcendance vers l'objet - ni la transcendance vers l'avenir - ni la transcendance vers l'amour - mais la transcendance de l'expression -59 .

Et la note suivante:

Non pas vers qqch. - mais l'extériorité même du sujet - l'être dans son être est expression - a une gloire qui le double. Le mystère c'est ce qqch. de plus que cette gloire et qui se refuse à cette gloire ${ }^{60}$.

Ces notes sont assez courtes et énigmatiques. Cela dit, il me semble qu'elles ne contredisent pas la supposition faite plus haut. Levinas n'envisage pas l'amour comme un but du mouvement de la transcendance, mais il dit que l'amour crée ce mouvement lui-même.

Quant à Rosenzweig, sa philosophie étant née à la fin de la Première Guerre mondiale et au commencement de la République de Weimar, elle semble pénétrée par l'optimisme et l'espoir. Rosenzweig semble croire que des conditions sociales sont déjà prêtes pour une mise en pratique de l'exécution du commandement de l'amour pour le prochain. La philosophie de Levinas a pris sa forme dans des conditions tout à fait différentes. L'optimisme de Rosenzweig est devenu intenable. Et plus tard on voit Levinas s'écarter pas à pas de la philosophie de l'amour de Franz Rosenzweig en continuant ses recherches sur la forme dans laquelle la présence de Dieu se manifeste. Ce problème devient de plus en plus difficile de par le pessimisme d'Emmanuel Levinas. L'histoire apparaît chez Levinas plutôt comme un cercle où des nouvelles épreuves et de nouvelles souffrances viennent toujours après une période d'accalmie, ce qui aggrave le problème de la présence de Dieu. On peut dire que dans toute son ouvre Levinas a cherché la présence divine dans la transcendance, dans l'au-delà. Cette structure fondamentale de la pensée lévinassienne a été fixée depuis le commencement, comme on le voit dans les Carnets. Ce que Levinas n'a pas encore découvert dans les Carnets, c'est la forme selon laquelle la transcendance se manifeste. Levinas a essayé et abandonné un certain nombre de voies. C'est dans cet aspect que sa philosophie a subi des changements profonds.

Quant à la théorie de l'eros, on voit que dans Totalité et Infini Levinas souligne la particularité de la relation sexuelle. Elle est tellement compliquée qu'il est impossible de la réduire à la relation éthique. Il est aussi

59. Ibid., p. 195 .

60. Ibid. 
impossible de la réduire au plaisir du plaisir de l'Autre et à la douleur de la douleur de l'Autre. Ce fait n'est pas dû à la complexité des rapports entre des partenaires sexuels. L'idée de Levinas diffère de certaines idées et associations contemporaines. Pour lui, le fait ontologique primordial est que la jouissance érotique vise la progéniture. Selon cet aspect, sa vision de l'eros est tout à fait proche de Rosenzweig, parce que tous les deux sont fidèles à la tradition du judaïsme. Voici une citation des Carnets où Levinas dit de sa philosophie qu'elle «suit le rythme du j. - car à travers paternité - sentiment généreux. Patriarches et leurs troupeaux - enfants - prophètes $"{ }^{6}$. On voit ici qu'en parlant du judaïsme Levinas dévoile le sens de la paternité. Donc la particularité de l'amour sexuel consiste en ce que par son amour même le sujet destine sa bien-aimée à être "mangée» par la progéniture. Elle peut être pour lui une fin en soi, mais par là elle deviendra le moyen pour la vie de leur progéniture.

Même si Levinas a transformé profondément la compréhension d'Autrui en cherchant toujours dans la relation à Autrui la manifestation de la transcendance, il semble cependant que l'idée d'Autrui conserve des traces de son origine dans la théorie de l'eros, que ce soient les descriptions du désir métaphysique ou bien celles de la proximité.

Zinaida SOKULER

Université d'État de Moscou

61. Carnets de captivité, p. 134. 